Sufrir y crecer con la enfermedad

\title{
Suffering and personal growth in coping with illness
}

https://doi.org/10.23938/ASSN.0747

M. Martínez, C. Centeno

En este número de Anales del Sistema Sanitario de Navarra se publican tres estudios diferentes que reflejan las distintas caras del sufrimiento ante la enfermedad. Uno de ellos trata sobre el sufrimiento del paciente que tiene dificultades para adaptarse al trasplante cardiaco ${ }^{1}$, otro versa sobre el sufrimiento de la familia (en este caso, de los padres de niños oncológicos ${ }^{2}$ ), y un tercer artículo aborda el sufrimiento del profesional, médico de Atención Primaria ${ }^{3}$. Sufre el enfermo, sufre su familia y puede sufrir el profesional que les atiende. Sin embargo, una lectura detenida de los trabajos nos permite ser optimistas porque se ve cómo la enfermedad puede ser también ocasión de crecimiento personal para los diferentes agentes implicados.

Sarasa y Olano-Lizarraga ${ }^{1}$ revisan veinticinco artículos sobre la experiencia de vivir con un corazón trasplantado. En el trabajo se pone de manifiesto cómo la medicina se ha centrado tradicionalmente en la prevención y tratamiento de las complicaciones físicas que pueden surgir tras el trasplante. Esto es así porque se considera un éxito que el paciente pueda continuar viviendo con mejor calidad y durante más tiempo, y no se ha planteado la importancia de tener en cuenta otros aspectos. Las autoras revisan esos otros aspectos, las complicaciones y repercusiones espirituales, psicológicas y sociales del trasplante, además de la relación con el donante y con el órgano.

Es evidente que en el caso del trasplante de corazón, además de las dificultades mencionadas, se añaden otros matices e implicaciones culturales relacionadas con el significado que se suele dar al corazón, como lugar que se asocia a los sentimientos, la personalidad o el alma.

Una enfermedad grave genera un impacto emocional que afecta al paciente y a su familia, en los que puede surgir un sentimiento de vulnerabilidad o fragilidad. Este sufrimiento que influye tanto a nivel físico como social, psicológico y/o espiritual tiene repercusiones en la calidad de vida del enfermo ${ }^{4,5}$. Además, puede dificultar el abordaje del paciente

Servicio de Medicina Paliativa. Clínica Universidad de Navarra. Pamplona.

Instituto Cultura y Sociedad. Universidad de Navarra. Pamplona.
Correspondencia:

Marina Martínez García

Servicio de Medicina Paliativa

Clínica Universidad de Navarra

Avda. Pío XII, 36

31008 Pamplona

E-mail: mmargarcia@unav.es 
al hacer más complicado el control de otros síntomas físicos o la adherencia a los tratamientos ${ }^{6,7}$.

Los profesionales sanitarios tenemos la responsabilidad de ir más allá de la esfera física del enfermo. Debemos ser capaces de desarrollar estrategias para evaluar a la persona enferma en su totalidad y para tener en cuenta esa otra parte del sufrimiento. Solo así seremos capaces de ayudarle en un proceso que además puede ser de crecimiento personal si, cuando se enfrenta a la dificultad, se siente comprendido y acompañado por el equipo que le atiende. En ese abordaje holístico se impone también una mirada a la familia.

López y col presentan un trabajo sobre el crecimiento postraumático en padres de niños y adolescentes con cáncer ${ }^{2}$. Definen el crecimiento postraumático como un conjunto de cambios positivos que ocurren como resultado de la lucha contra una crisis o desafío. El crecimiento postraumático supone una mejora o un desarrollo en la persona respecto al estado previo al suceso traumático. En la revisión que llevan a cabo sobre veinte estudios muestran que los padres de niños con cáncer tienen un crecimiento postraumático mayor que los de niños sanos.

La enfermedad de un hijo, siendo niño o adolescente, es una de las situaciones más duras que pueden atravesar sus progenitores. La literatura abunda en el impacto y el sufrimiento negativo de la enfermedad en los padres cuando uno de sus hijos enferma. Estudios en esta línea muestran que a lo largo de la enfermedad oncológica infantil es frecuente en los padres la aparición de sentimientos de vulnerabilidad, tristeza, temor, depresión, ansiedad, pánico y aislamiento social ${ }^{8}$.

Ante estos estudios nos podemos preguntar si es posible mirar más allá del sufrimiento y la desesperación cuando se tiene un hijo gravemente enfermo. ¿Se puede crecer personalmente en una situación de este tipo?

Numerosos estudios intentan explicar las diferencias entre los pacientes y familiares que saben desarrollar un estilo de afrontamiento que les permite enfrentarse a la adversidad de una manera más adaptativa y los que no. Algunos trabajos estudian intervenciones basadas en la búsqueda de sentido o en el aumento de la percepción de dignidad del paciente y de la familia como maneras de crecimiento personal que facilitan hacer frente a un sufrimiento extremo como puede ser la muerte cercana ${ }^{9,10}$.

La revisión que aquí se presenta muestra que es posible ese crecimiento personal en medio del sufrimiento por la enfermedad del hijo ${ }^{2}$. Esto no quiere decir que estos padres no sufran. A diferencia de lo que puede parecer, este crecimiento se produce a la vez que la persona intenta adaptarse a las circunstancias negativas, y puede coexistir con el sufrimiento y el malestar psicológico.

Por último, también los profesionales sufrimos ante la enfermedad. Trabajar diariamente con pacientes y sus familias puede provocar dificultades en el profesional que conducen a problemas graves como el síndrome de burnout. En ocasiones, unas expectativas irreales y los sentimientos de impotencia, junto con el exceso de trabajo o las dificultades institucionales, pueden ser los detonantes de ese agotamiento emocional que, junto con la despersonalización y una reducida realización personal, forman el síndrome de burnout ${ }^{11}$. 
En este número también se aborda un trabajo de Fuertes y col sobre la persistencia a largo plazo de los efectos de un programa de mindfulness y autocompasión en profesionales sanitarios de Atención Prima$\mathrm{ria}^{3}$. Los autores presentan un estudio prospectivo con cuarenta y ocho profesionales que, con un programa de dos horas y media por semana durante ocho semanas, mejoran significativamente en autocompasión y en el estrés que perciben, manteniéndose esta mejoría a los dos años. Sin embargo, no encontraron diferencias significativas en el nivel de burnout.

Es posible que el burnout requiera de una intervención más holística sobre el profesional. Probablemente precisa un trabajo integral con el profesional, que comience por el autoconocimiento y la conciencia de la propia vulnerabilidad, seguido de un entrenamiento específico en estrategias de afrontamiento personal. En definitiva se trata de que, como profesionales, sepamos crecer también ante el sufrimiento de aquellos a los que cuidamos.

En conclusión, un enfoque holístico en la atención al enfermo y a la familia no solo alivia al enfermo. Todos, paciente, familia y profesional sufrimos y podemos crecer en el proceso de enfermedad cuando intentamos el abordaje integral.

\section{BIBLIOGRAFÍA}

1. Sarasa MM, Olano-Lizarraga M. Explorando la experiencia de vivir con un trasplante cardíaco: una revisión sistemática de la literatura. An Sist Sanit Navar 2019; 42: 309-324. https://doi.org/10.23938/ASSN.0719

2. López J, ORTiz G, Noriega C. Crecimiento postraumático en padres de niños y adolescentes con cáncer. An Sist Sanit Navar 2019; 42: 325-337. https://doi. org/10.23938/ASSN.0717

3. Fuertes C, Aranda G, Rezola N, Erramuzpe A, Palacios C, Ibáñez B. Persistencia a largo plazo de los efectos de un programa de mindfulness y autocompasión en profesionales sanitarios de Atención Primaria. An Sist Sanit Navar 2019; 42: 269-280. https://doi.org/10.23938/ASSN.0718

4. Wilson KG, Chochinov HM, McPherson CJ, LeMay K, Allard P, Chary S et al. Suffering with advanced cancer. J Clin Oncol 2007; 25: 1691-1697. https://doi. org/10.1200/jco.2006.08.6801

5. Kissane DW, Clarke DM, Street AF. Demoralization syndrome: a relevant psychiatric diagnosis for palliative care. J Palliat Care 2001; 17: 12-21. https:// doi.org/10.1177/082585970101700103

6. Fainsinger RL, Nekolaichuk CL, Lawlor PG, Neumann CM, Hanson J, Vigano A. A multicenter study of the revised Edmonton Staging System for classifying cancer pain in advanced cancer patients. J Pain Symptom Manage 2005; 29: 224-237. https://doi.org/10.1016/j.jpainsymman.2004.05.008

7. Lloyd-Williams M, Dennis M, Taylor F. A prospective study to determine the association between physical symptoms and depression in patients with advanced cancer. Palliat Med 2004; 18: 558-563. https://doi. org/10.1191/0269216304pm923oa

8. National Comprehensive Cancer Network. NCCN Clinical practice guidelines in oncology 2012. https://www.nccn.org/professionals/physician_gls/

9. BREITBART W. Spirituality and meaning in supportive care: spirituality and meaning-centered group psychotherapy interventions in advanced cancer. Support Care Cancer 2002; 10: 272-280. https://doi.org/10.1007/s005200100289 
10. Chochinov HM, Hack T, Hassard T, Kristjanson LJ, McClement S, Harlos M. Dignity therapy: a novel psychotherapeutic intervention for patients near the end of life. J Clin Oncol 2005; 23: 5520-5525. https://doi.org/10.1200/jco.2005.08.391

11. MASLACH, C. JACKSON, S.E. Burnout in health professions: A social psychological analysis. En: Sanders G, Suls J, editores. Social psychology of health and illness. Hillsdale: Erlbaum, 1982. 